\title{
The Maxwell - Exponential Distribution: Theory and Application to Lifetime Data
}

\author{
Usman Aliyu Abdullahi ${ }^{1}$, Ahmad Abubakar Suleiman ${ }^{1}$, Aliyu Ismail Ishaq ${ }^{2}$, Abubakar Usman $^{2}$ and
} Aminu Suleiman ${ }^{1}$

${ }^{I}$ Department of Statistics, Faculty of Computing and Mathematical Science, Kano University of Science and Technology Wudil, Nigeria

${ }^{2}$ Department of Statistics, Faculty of Physical Science, Ahmadu Bello University Zaria, Nigeria *Corresponding author: usiyamma@gmail.com

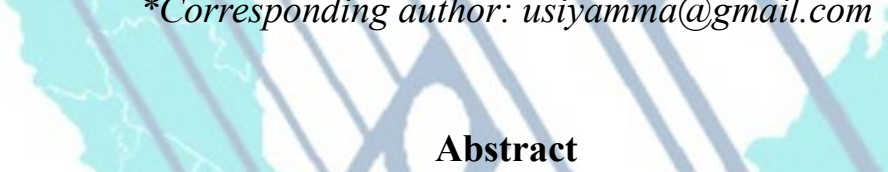

Two parameters Maxwell - Exponential distribution was proposed using the Maxwell generalized family of distribution. The probability density function, cumulative distribution function, survival function, hazard function, quantile function, and statistical properties of the proposed distribution are discussed. The parameters of the proposed distribution have been estimated using the maximum likelihood estimation method. The potentiality of the estimators was shown using a simulation study. The overall assessment of the performance of Maxwell - Exponential distribution was determined by using two real-life datasets. Our findings reveal that the Maxwell - Exponential distribution is more flexible compared to other competing distributions as it has the least value of information criteria.

Keywords: Maxwell - Exponential, Maxwell generalized family, maximum likelihood estimator and Quantile function.

\section{Introduction}

The real lifetime datasets are so enormous to the extent that the existence lifetime distributions that are used in modeling lifetime datasets are not enough to handle all the present data (Suleiman et al., 2020). These prompt scientists from all fields working tirelessly to proposed new distributions that will be used in modeling present and past datasets, by improving the flexibility of the new distributions that were derived from an existing family of distributions.

There are various approaches used in proposing lifetime distributions as studied by many researchers (Alzaatreh et al., 2013; Bourguignon et al., 2014). Several researchers used these methods to propose new distribution such as (Yousof et al., 2016; Alizadeh et al., 2017; Cordeiro et al., 2017; Gomes et al., 2017; Jamal et al., 2017; Ahsan U1 Haq et al., 2018; Muhammad et al., 2018; Nadia and Lamyaa, 2018).

Weibull and Gamma distributions are the most widely two parametric distributions for analyzing most of the lifetime datasets (Weibull W. 1939; Johnson et al., 1994), it also has more application apart 
from the modeling of lifetime data (Klinken J. and Van A., 1961; Alexander G. N., 1962; Jackson O. A. Y., 1963). The Gamma distribution with two parameters namely scale and shape parameters is more flexible to model real positive data, it's shape parameter enable it to possess increasing and decreasing failure rate but it's little drawback is that it has incomplete gamma function which makes it to be more complicated in expressing its survival function and hazard function. This reason makes Weibull distribution more attractive because of its well defined statistical properties (Gupta and Kundu, 2001). Maxwell introduced Maxwell distribution which is popularly known for modeling data related to science. It can also be used to model positively skewed datasets. But its great failure is incapable to model lifetime dataset which is skewed to the left or right due to its increasing failure rate (Ishaq and Abiodun, 2020). Suppose random variable $X$ follows Maxwell distribution, then its cdf is given as follows;

$$
F(x, \beta)=\frac{2}{\sqrt{\pi}} \gamma\left(\frac{3}{2}, \frac{x^{2}}{2 \beta^{2}}\right), \beta>0, x \geq 0
$$

where $\gamma\left(\frac{3}{2}, \frac{x^{2}}{2 \beta^{2}}\right)$ is incomplete gamma function and is defined as for $x>0$ and $a>0$, $\gamma(a, x)=\int_{0}^{x} t^{a-1} e^{-t} d t$

and its corresponding probability density function is defined by;

$$
f(x, \beta)=\sqrt{\frac{2}{\pi}} \frac{x^{2} e^{-\frac{x^{2}}{2 \beta^{2}}}}{\beta^{3}}, \beta>0, x \geq 0
$$

where $\beta$ is a scale parameter.

Exponential distribution is receiving considerable attention, because many scientists used it as their baseline distribution while others remodel it to another distribution. For example, Gupta introduced Exponentiated Exponential distribution (Gupta and Kundu, 2001), it consists of raising power to the cumulative function of exponential distribution by positive parameter which make it to two parameters distribution. Its shape parameter enables it to possess increasing or decreasing failure rate. Oguntunde extended Exponential distribution and proposed Weibull - Exponential distribution (Oguntunde et al., 2015).

Ishaq proposed Maxwell generalized family of distributions (Ishaq and Abiodun, 2020). The Maxwell generalized family is later extended to Maxwell - Weibull distribution. The cumulative distribution and probability density functions of Maxwell generalized family of distributions is respectively given as;

$$
F(x, \lambda, \beta)=\frac{2}{\sqrt{\pi}} \gamma\left(\frac{3}{2}, \frac{1}{2 \beta^{2}}\left(\frac{M(x, \xi)}{1-m(x, \xi)}\right)^{2}\right), \beta>0, x \in \mathbb{R}
$$




$$
F(x, \lambda, \beta)=\frac{2 m(x, \xi)}{\beta^{2} \sqrt{2 \pi}(1-M(x, \xi))^{2}}\left(\frac{M(x, \xi)}{1-m(x, \xi)}\right)^{2} \exp \left(-\frac{1}{2 \beta^{2}}\left(\frac{M(x, \xi)}{1-m(x, \xi)}\right)^{2}\right)
$$

where $M(x, \xi), m(x, \xi)$ and $\xi$ are the cumulative distribution function, probability density function and parameter of the baseline distribution respectively. A new class of Exponential distribution is introduced by applying Maxwell generalized family of distributions.

The method used in proposing the Maxwell generalized family of distribution was introduced by Alzaatreh (Alzaatreh et al., 2013). The cumulative distribution function of a new generalized family of distribution is given by;

$$
F(x)=\int_{c}^{N(M(x))} f(x) d x \quad R \subset[c, k] ; \quad-\infty \leq c<k \leq \infty
$$

where $N(M(x))$ is the link function of distribution function $M(x)$ for any random variable $X$ and $f(x)$ is the density function of random variable $R$.

\section{The Cumulative Distribution and Probability Density Functions of Maxwell - Exponential}

\section{Distribution}

The section introduces the Maxwell - Exponential $\left(\mathrm{M}_{\mathrm{wl}} \mathrm{E}\right)$ distribution. The motivation behind this research is to obtain flexible distribution that can provide different shapes of the density and hazard functions, and also to provide a great flexibility when tested against its competing models from wellknown family of distributions.

The cumulative distribution and probability density functions of $\mathrm{M}_{\mathrm{wl}}-\mathrm{E}$ distribution can be obtained by inserting the cumulative distribution and probability density functions of Exponential distribution in to equations (3) and (4) respectively.

$$
\begin{aligned}
& F(x, \lambda, \beta)=\frac{2}{\sqrt{\pi}} \gamma\left(\frac{3}{2}, \frac{1}{2 \beta^{2}}\left(\frac{1-e^{-\lambda x}}{e^{-\lambda x}}\right)^{2}\right), \lambda, \beta, x>0 \\
& f(x, \lambda, \beta)=\frac{2 \lambda e^{-\lambda x}}{\beta^{2} \sqrt{2 \pi}\left(e^{-\lambda x}\right)^{2}}\left(\frac{1-e^{-\lambda x}}{e^{-\lambda x}}\right)^{2} \exp \left(-\frac{1}{2 \beta^{2}}\left(\frac{1-e^{-\lambda x}}{e^{-\lambda x}}\right)^{2}\right)
\end{aligned}
$$

where $\beta$ and $\lambda$ are the scale parameters. For more simplicity let the cumulative density function be written as $F(x)=F(x, \lambda, \beta)$ and the probability density function $f(x)=f(x, \lambda, \beta)$ throughout the paper.

It can be observed from the figure 1 below, the plot of Maxwell - Exponential distribution possess right and left skewed pattern. Therefore, the Maxwell - Exponential distribution can fit data set that has both right and left-skewed. 

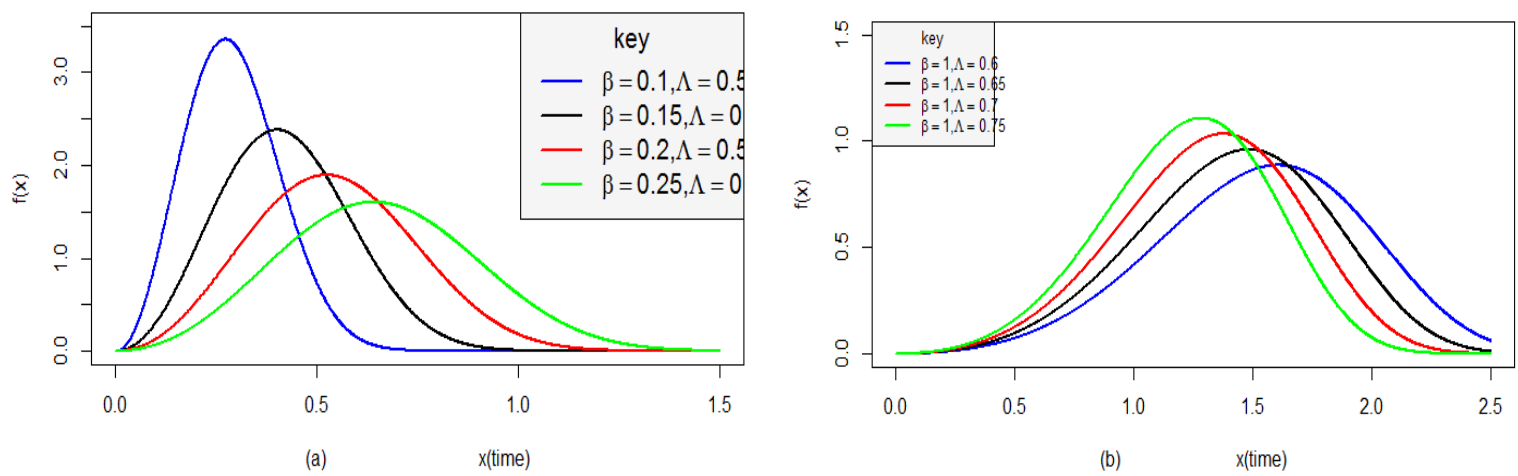

Figure 1. Plot of Probabilty density function of $\mathrm{M}_{\mathrm{wl}}-\mathrm{E}$ distribution at the various values of parameters.

\section{An Important Linear Representation}

Linear representation of $\mathrm{M}_{\mathrm{wl}} \mathrm{E}$ density function will be provided here, by applying the Exponential power series expansion to last term of equation (6) for $x>0$

$$
e^{-x}=\sum_{i=0}^{\infty} \frac{(-1)^{i}}{i !} x^{i}
$$

Equation (6) now becomes

$$
f(x)=\frac{2 \lambda}{2^{i} \beta^{3+2 i} \sqrt{2 \pi}} \sum_{i=0}^{\infty} \frac{(-1)^{i}}{i !} e^{-\lambda x} \frac{\left(1-e^{-\lambda x}\right)^{2+2 i}}{\left[1-\left(1-e^{-\lambda x}\right)\right]^{4+2 i}}
$$

Now consider the expansion for $|z|<1, b>0$, then the power series is defined to be

$$
(1-z)^{-b}=\sum_{j=0}^{\infty} \frac{\Gamma(b+j)}{j ! \Gamma(b)} z^{j}
$$

equation (7) can be further rewritten as

$$
f(x)=\frac{2 \lambda}{2^{i} \beta^{3+2 i} \sqrt{2 \pi}} \sum_{i, j=0}^{\infty} \frac{(-1)^{i}}{i ! j !} \frac{\Gamma(4+2 i+j)}{\Gamma(4+2 i)} e^{-\lambda x}\left(1-e^{-\lambda x}\right)^{2+2 i+j}
$$

Now consider the series of generalized binomial expansion

$$
(1-z)^{b-1}=\sum_{k=0}^{\infty} \frac{(-1)^{k} \Gamma(b)}{k ! \Gamma(b-k)} z^{k} \text { for }|z|<1, b>0
$$

Equation (8) can further be simplified by applying (9) as

$$
f(x)=\sum_{k=0}^{\infty} D_{(k)} e^{-\lambda(1+k) x}
$$


where $D_{(k)}=\frac{2 \lambda}{2^{i} \beta^{3+2 i} \sqrt{2 \pi}} \sum_{i, j=0}^{\infty} \frac{(-1)^{i}}{i ! j !} \frac{\Gamma(4+2 i+j)}{\Gamma(4+2 i)} e^{-\lambda x}\left(\begin{array}{c}2+2 i+j \\ k\end{array}\right)$.

\section{The Survival and Hazard Functions of the Maxwell - Exponential Distribution}

Here survival and hazard functions of $\mathrm{M}_{\mathrm{wl}}-\mathrm{E}$ distribution was described.

\subsection{The Survival Function}

The survival function $(S(x))$ For $\mathrm{M}_{\mathrm{wl}}$-E distribution were obtained as

$$
S(x)=1-\frac{2}{\sqrt{\pi}} \gamma\left(\frac{3}{2}, \frac{1}{2 \beta^{2}}\left(\frac{1-e^{-\lambda x}}{e^{-\lambda x}}\right)^{2}\right)
$$

where $\beta>0$ and $\lambda>0$ are scale parameters.

\subsection{The Hazard Function}

The hazard function $(h(x))$ for $\mathrm{M}_{\mathrm{wl}}-\mathrm{E}$ distribution were obtained as

$$
h(x)=\frac{\frac{2 \lambda e^{-\lambda x}}{\left(e^{-\lambda x}\right)^{2}}\left(\frac{1-e^{-\lambda x}}{e^{-\lambda x}}\right)^{2} \exp \left(-\frac{1}{2 \beta^{2}}\left(\frac{1-e^{-\lambda x}}{e^{-\lambda x}}\right)^{2}\right)}{\beta^{3} \sqrt{2 \pi}\left\{1-\frac{2}{\sqrt{\pi}} \gamma\left(\frac{3}{2}, \frac{1}{2 \beta^{2}}\left(\frac{1-e^{-\lambda x}}{e^{-\lambda x}}\right)^{2}\right)\right\}}
$$

It can be observed from figure 2 below that, the hazard function of Maxwell - Exponential distribution has increasing function.

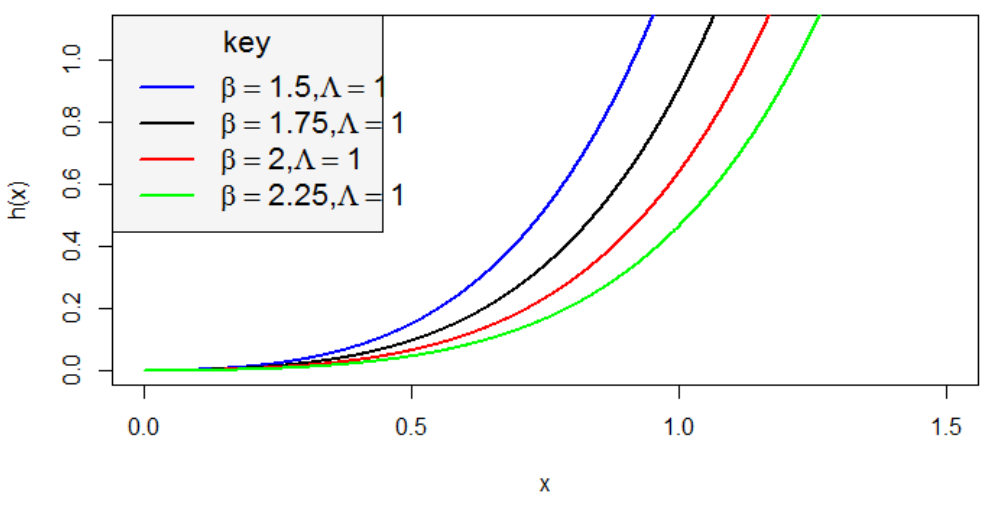

Figure 2. The Plot of hazard function of $M_{w l}-E$ distribution 


\section{The Basic Properties of Maxwell - Exponential Distribution}

The basic properties of $\mathrm{M}_{\mathrm{wl}} \mathrm{E}$ distribution namely; the moments, incomplete moments, probability weighted moments and order statistics are presented here.

\subsection{The Moments}

Suppose random variable $X$ follows $\mathrm{M}_{\mathrm{wl}} \mathrm{E}$ distribution, then the non - central moment of $X$ is given by;

$$
E\left(x^{r}\right)=\int_{-\infty}^{\infty} x^{r} f(x) d x
$$

Here $f(x)$ is the probability density function of $\mathrm{M}_{\mathrm{wl}}-\mathrm{E}$ distribution, inserting equation (10) into equation (13) gives;

$$
E\left(x^{r}\right)=\sum_{k=0}^{\infty} D_{(k)} \int_{0}^{\infty} x^{r} e^{-\lambda(1+k) x} d x
$$

Let $y=\lambda(1+k) x \Rightarrow x=\frac{y}{\lambda(1+k)}$ and $d x=\frac{d y}{\lambda(1+k)}$

By substituting equation (15) into equation (14) we have;

$$
\begin{aligned}
E\left(x^{r}\right) & =\sum_{k=0}^{\infty} D_{(k)} \int_{0}^{\infty}\left(\frac{y}{\lambda(1+k)}\right)^{r} e^{-y} \frac{d y}{\lambda(1+k)} \\
& =\sum_{k=0}^{\infty} D_{(k)} \frac{1}{(\lambda(1+k))^{r+1}} \int_{0}^{\infty} y^{r+1-1} e^{-y} d y \\
& =\sum_{k=0}^{\infty} D_{(k)} \frac{\Gamma(r+1)}{(\lambda(1+k))^{r+1}}
\end{aligned}
$$

Equation (17) gives moments of the $\mathrm{M}_{\mathrm{wl}}-\mathrm{E}$ distribution.

\subsection{The Incomplete Moments}

The incomplete moments of $\mathrm{M}_{\mathrm{wl}} \mathrm{E}$ distribution denoted by $\phi_{r}(t)$ is defined by;

$$
\phi_{r}(t)=\int_{-\infty}^{t} x^{r} f(x) d x
$$

Here $f(x)$ is the pdf of $\mathrm{M}_{\mathrm{wl}}-\mathrm{E}$ distribution defined in equation (10).

$$
\phi_{r}(t)=\sum_{k=0}^{\infty} D_{(k)} \int_{0}^{t} x^{r} e^{-\lambda(1+k) x} d x
$$


By substituting equation (15) into equation (19) we have;

$$
\begin{aligned}
\phi_{r}(t) & =\sum_{k=0}^{\infty} D_{(k)} \int_{0}^{\lambda(1+k) t}\left(\frac{y}{\lambda(1+k)}\right)^{r} e^{-y} \frac{d y}{\lambda(1+K)} \\
& =\sum_{k=0}^{\infty} D_{(k)} \frac{1}{(\lambda(1+k))^{r+1}} \int_{0}^{\lambda(1+k) t} y^{r} e^{-y} d y \\
& =\sum_{k=0}^{\infty} D_{(k)} \frac{1}{(\lambda(1+k))^{r+1}} \gamma(r+1, \lambda(1+k) t)
\end{aligned}
$$

Equation (21) is incomplete moments of the $\mathrm{M}_{\mathrm{wl}}-\mathrm{E}$ distribution.

\subsection{The Probability Weighted Moments}

The probability weighted moments of $r, q \in R$, and $z=0$ is defined below;

$$
\rho(r, s, o)=E\left\{X^{s} F(X)^{r}\right\}=\int_{-\infty}^{\infty} x^{s} F(x)^{r} f(x) d x
$$

Where $F(x)$ and $f(x)$ are given in equation (5) and equation (6) respectively. Thus;

$$
F(x)^{r} f(x)=\lambda \sum_{b, i, j, k, l=0}^{\infty} Q_{(b, i, j, k, l)} e^{-\lambda x}\left(1-e^{-\lambda x}\right)^{2+2 i+3 b+2 k+l}
$$

where $Q_{(b, i, j, k, l)}=\frac{(-1)^{k} B_{a}(r) A_{b, i} \Gamma(4+2 i+3 b+2 k+l)}{\beta^{3+2 i+3 b+2 k} 2^{2 i+3 b+k}\left(\Gamma\left(\frac{3}{2}\right)\right)^{b} k ! l ! \Gamma(4+2 i+3 b+2 k)} \sqrt{\frac{2}{\pi}}$

$$
\begin{aligned}
& B_{a}(r)=\sum_{l=b}^{\infty}(-1)^{l+m}\left(\begin{array}{l}
r \\
l
\end{array}\right)\left(\begin{array}{l}
l \\
m
\end{array}\right) \\
& A_{b, i}=\left(i d_{o}\right)^{-1} \sum_{j=1}^{i}\left(b_{j}-i+j\right) d_{j} \text { and } d_{j}=\frac{(-1)^{j}}{j !(m+j)} .
\end{aligned}
$$

Then by substituting equation (23) into equation (22) gives;

$$
\rho_{(r, s, o)}=\lambda \sum_{b, i, j, k, l=0}^{\infty} Q_{(b, i, j, k, l)} \int_{0}^{\infty} x^{r} e^{-\lambda x}\left(1-e^{-\lambda x}\right)^{2+2 i+3 b+2 k+l} d x
$$

Applying generalized binomial expansion as defined in equation (9) into equation (24) we have;

$$
\rho_{(r, s, o)}=\lambda \sum_{b, i, j, k, l, m=0}^{\infty} Q_{(b, i, j, k, l)}(-1)^{m}\left(\begin{array}{c}
2+2 i+3 b+2 k+l \\
m
\end{array}\right) \int_{0}^{\infty} x^{r} e^{-\lambda(1+m) x} d x
$$


Let $t=\lambda(1+m) x \Rightarrow x=\frac{t}{\lambda(1+m)}$ and $d x=\frac{d t}{\lambda(1+m)}$

By substituting equation (26) into equation (25) gives;

$$
\begin{aligned}
\rho_{(r, s, o)} & =\lambda \sum_{b, i, j, k, l, m=0}^{\infty} Q_{(b, i, j, k, l)}(-1)^{m}\left(\begin{array}{c}
2+2 i+3 b+2 k+l \\
m
\end{array}\right) \int_{0}^{\infty}\left(\frac{t}{\lambda(1+m)}\right)^{r} \frac{d t}{\lambda(1+m)} \\
& =\lambda \sum_{b, i, j, k, l, m=0}^{\infty} Q_{(b, i, j, k, l)}(-1)^{m} \frac{(-1) \Gamma(r+1)}{(\lambda(1+m))^{r+1}}\left(\begin{array}{c}
2+2 i+3 b+2 k+l \\
m
\end{array}\right)
\end{aligned}
$$

Equation (27) is probability weighted moments of $\mathrm{M}_{\mathrm{wl}}-\mathrm{E}$ distribution.

\subsection{The Order Statistics}

Let $X_{k}(k=1, \ldots, n)$ denote $\mathrm{M}_{\mathrm{wl}}-\mathrm{E}$ random variable with samples size $n$, then the density function of the $k^{\text {th }}$ order statistics of $\mathrm{M}_{\mathrm{wl}}-\mathrm{E}$ distribution denoted by $f_{k, n}(x)$ is given as;

$$
f_{k, n}(x)=\frac{n !}{(k-1) !(n-k) !} f(x) F(x)^{k-1}[1-F(x)]^{n-1}
$$

where $F(x)$ and $f(x)$ are defined in equation (5) and equation (6) respectively.

But

$$
F(x)^{k-1}[1-F(x)]^{n-1}=\sum_{p=0}^{\infty}(-1)^{p}\left(\begin{array}{c}
n-k \\
p
\end{array}\right) F(x)^{k+p-1}
$$

By substituting equation (29) into equation (28) we have;

$$
f_{k, n}(x)=\frac{n !}{(k-1) !(n-k) !} \sum_{p=0}^{\infty}(-1)^{p}\left(\begin{array}{c}
n-k \\
p
\end{array}\right) F(x)^{k+p-1}
$$

Now, $f(x) F(x)^{k+p-1}$ is defined for $s=k+p-1$ from equation (23). Thus,

$$
\begin{array}{r}
f_{k, n}(x)=\lambda \sum_{m, i, j, k, l, p=0}^{\infty} \frac{(-1)^{p+q} n ! Q_{(b, i, j, k, l)}}{(k-1) !(n-k) !}\left(\begin{array}{c}
n-k \\
p
\end{array}\right) e^{-\lambda x}\left(1-e^{-\lambda x}\right)^{2+2 i+3 b+2 k+l} \\
f_{k, n}(x)=\lambda \sum_{m, i, j, k, l, p=0}^{\infty} \frac{(-1)^{p+q} n ! Q_{(b, i, j, k, l)}}{(k-1) !(n-k) !}\left(\begin{array}{c}
n-k \\
p
\end{array}\right)\left(\begin{array}{c}
2+2 i+3 b+2 k+l \\
n
\end{array}\right) e^{-\lambda(1+p) x}
\end{array}
$$

\section{Parameter Estimation}

Let $X_{t}(t=1, \ldots, n)$ denote a random sample from $\mathrm{M}_{\mathrm{wl}} \mathrm{E}$ with parameters $\lambda$ and $\beta$. By using the maximum likelihood estimation method, the likelihood function gives; 


$$
f\left(x_{t}(t=1, \ldots n), \lambda, \beta\right)=\prod_{i=1}^{n}\left[\frac{2 \lambda e^{-\lambda x}}{\beta^{3} \sqrt{2 \pi}\left(e^{-\lambda x}\right)^{2}}\left(\frac{1-e^{-\lambda x}}{e^{-\lambda x}}\right)^{2} \exp \left(-\frac{1}{2 \beta^{2}}\left(\frac{1-e^{-\lambda x}}{e^{-\lambda x}}\right)^{2}\right)\right]
$$

Let $l=\log f\left(x_{t}(t=1, \ldots n), \lambda, \beta\right)$ denote the $\log -$ likelihood function then,

$$
\begin{aligned}
& l=n \log (2)+n \log (\lambda)-\lambda \sum_{t=1}^{n} x_{t}-3 n \log (\beta)-\frac{n}{2} \log (2 \pi)-2 \sum_{t=1}^{n} \log \left[\mathrm{e}^{-\lambda x_{t}}\right]+ \\
& 2 \sum_{t=1}^{n} \log \left[\frac{1-e^{-\lambda x_{t}}}{e^{-\lambda x_{t}}}\right]-\frac{1}{2 \beta^{2}} \sum_{t=1}^{n}\left[\frac{1-e^{-\lambda x_{t}}}{e^{-\lambda x_{t}}}\right]^{2}
\end{aligned}
$$

The maximum likelihood estimated parameters can be obtained by differentiating $l$ with respect to parameters $\lambda$ and $\beta$ and equating to zero.

$$
\begin{aligned}
\frac{d l}{d \beta} & =-\frac{3 n}{\beta}+\frac{1}{\beta^{3}} \sum_{t=1}^{n}\left(\frac{1-e^{-\lambda x_{t}}}{e^{-\lambda x_{t}}}\right)^{2}=0 \\
& \Rightarrow-3 n \beta^{2}+\sum_{t=1}^{n}\left(\frac{1-e^{-\lambda x_{t}}}{e^{-\lambda x_{t}}}\right)^{2}=0 \\
& \Rightarrow \hat{\beta}=\sqrt{\frac{1}{3 n} \sum_{t=1}^{n}\left(\frac{1-e^{-\lambda x_{t}}}{e^{-\lambda x_{t}}}\right)^{2}}
\end{aligned}
$$

Equation (35) is the maximum likelihood estimate of the parameter $\beta$

$$
\frac{d l}{d \lambda}=\frac{n}{\lambda}+\sum_{t=1}^{n} x_{t}+2 \sum_{t=1}^{n}\left(\frac{x_{t}}{1-e^{-\lambda x_{t}}}\right)-\frac{1}{\beta^{2}} \sum_{t=1}^{n}\left(\frac{1-e^{-\lambda x_{t}}}{e^{-\lambda x_{t}}}\right)\left(\frac{x_{t}}{e^{-\lambda x_{t}}}\right)=0
$$

Substituting equation (35) into (36) and solving the equation will yield the maximum likelihood estimate of the parameter $\lambda$, statistical software could be used to solve the equation.

\section{Applications}

This section described a simulation study using quantile function and application to real - life datasets to demonstrate the flexibility of $\mathrm{M}_{\mathrm{wl}}-\mathrm{E}$ distribution.

\subsection{The Quantile Function}

We obtained the quantile function of $\mathrm{M}_{\mathrm{wl}}$-E distribution by inverting its cumulative distribution function as given in equation (5).

$$
F(x, \lambda, \beta)=\frac{2}{\sqrt{\pi}} \gamma\left(\frac{3}{2}, \frac{1}{2 \beta^{2}}\left(\frac{1-e^{-\lambda x}}{e^{-\lambda x}}\right)^{2}\right)
$$


The cumulative distribution function can be express as;

$$
F(x, \lambda, \beta)=\frac{\gamma(n, m)}{\Gamma(n)}
$$

where $n=\frac{3}{2}$ and $m=\frac{1}{2 \beta^{2}}\left(\frac{1-e^{-\lambda x}}{e^{-\lambda x}}\right)^{2}$

The quantile function of the proposed $\mathrm{M}_{\mathrm{wl}}-\mathrm{E}$ model can be obtained from equation (37) below (Oluyede B, 2018).

$$
m=\gamma^{-1}(n, u \Gamma(n))
$$

By substituting for $n$ and $m$ equation (39) becomes;

$$
\frac{1}{2 \beta^{2}}\left(\frac{1-e^{-\lambda x}}{e^{-\lambda x}}\right)^{2}=\gamma^{-1}\left(\frac{3}{2}, u \Gamma\left(\frac{3}{2}\right)\right)
$$

This can be written as;

$$
\left(\frac{1-e^{-\lambda x}}{e^{-\lambda x}}\right)=\left\{2 \beta^{2} \gamma^{-1}\left(\frac{3}{2}, u \Gamma\left(\frac{3}{2}\right)\right)\right\}^{\frac{1}{2}}
$$

This can be express as,

$$
\frac{w}{1-w}=z
$$

where $w=1-e^{-\lambda x}$ and $z=\left\{2 \beta^{2} \gamma^{-1}\left(\frac{3}{2}, u \Gamma\left(\frac{3}{2}\right)\right)\right\}^{\frac{1}{2}}$

Now $w$ is given as;

$$
w=\frac{z}{1+z}
$$

Substituting $w$ in equation (44) becomes;

$$
\begin{aligned}
1-e^{-\lambda x} & =\frac{z}{1+z} \\
e^{-\lambda x} & =1-\left(\frac{z}{1+z}\right)
\end{aligned}
$$

Taking $\log$ to both sides of the equation gives;

$$
\lambda x=\log \left(1-\left(\frac{z}{1+z}\right)\right)
$$


The quantile function of the proposed $\mathrm{M}_{\mathrm{wl}}-\mathrm{E}$ distribution is given as;

$$
\begin{aligned}
& x=-\frac{1}{\lambda} \log \left(1-\left(\frac{z}{1+z}\right)\right) \\
& x=-\frac{1}{\lambda} \log \left(1-\left(\frac{\left\{2 \beta^{2} \gamma^{-1}\left(\frac{3}{2}, u \Gamma\left(\frac{3}{2}\right)\right)\right\}^{\frac{1}{2}}}{1+\left\{2 \beta^{2} \gamma^{-1}\left(\frac{3}{2}, u \Gamma\left(\frac{3}{2}\right)\right)\right\}^{\frac{1}{2}}}\right)\right)
\end{aligned}
$$

Equation (49) is the quantile of the proposed $\mathrm{M}_{\mathrm{wl}}-\mathrm{E}$ distribution.

\subsection{Simulation}

The simulation of the random numbers is based on the quantile function defined in equation (36), data were generated for different sample size $(n)$ starting from 20, 50, 80, 120, 320, 720 and 1120 at different values of parameters, by fixing $\beta=3.5$ and $\lambda=5.2$. After repeating the simulation 1000 times, the mean (M), variance (Var) and mean squared error (MSE) was obtained. It can be observed that in Table (1). The mean estimate for each parameter is approaching its fixed value.

Table 1. The performance of the $\left(\mathrm{M}_{\mathrm{wl}}-\mathrm{E}\right)$ distribution simulation

\begin{tabular}{|c|c|c|c|}
\hline Sample Size $(n)$ & Mean $(\mathrm{M})$ & Variance (Var) & MSE \\
\hline \multirow{2}{*}{20} & $\beta=5.594546$ & 23.954450 & 28.341570 \\
& $\lambda=5.765681$ & 2.3213180 & 2.6413140 \\
\hline \multirow{2}{*}{50} & $\beta=4.112448$ & 3.3493340 & 3.7244270 \\
& $\lambda=5.398237$ & 0.8226398 & 0.8619376 \\
\hline \multirow{2}{*}{80} & $\beta=3.874757$ & 1.6565820 & 1.7970240 \\
& $\lambda=5.335095$ & 0.4208483 & 0.4890990 \\
\hline \multirow{2}{*}{120} & $\beta=3.760524$ & 1.0262830 & 1.0941560 \\
& $\lambda=5.299490$ & 0.3165021 & 0.3264000 \\
\hline \multirow{2}{*}{320} & $\beta=3.605025$ & 0.3470734 & 0.3581035 \\
& $\lambda=5.241440$ & 0.1195623 & 0.1212795 \\
\hline \multirow{2}{*}{720} & $\beta=3.544303$ & 0.1396096 & 0.1415724 \\
& $\lambda=5.217804$ & 0.0514281 & 0.0517451 \\
\hline \multirow{2}{*}{1120} & $\beta=3.532054$ & 0.0872496 & 0.0882771 \\
& $\lambda=5.213228$ & 0.0322500 & 0.0324250 \\
\hline
\end{tabular}




\subsection{Model Comparison}

The proposed $\mathrm{M}_{\mathrm{wl}}-\mathrm{E}$ distribution will be compared with other distributions using real - life datasets to demonstrate its flexibility. The competing models are; Exponential distribution, Exponentiated Exponential distribution and Burr $\mathrm{X}$ - Exponential distribution. The competing models will be represented as $E_{x p}-X, E_{x p}-E$ and $B_{u x}-E$ respectively. The first competing model has only scale parameter. It is also the baseline distribution of the proposed model research. The second and third models have two parameters (scale and shape parameters). The distribution functions of the competitive models are respectively given as;

$$
\begin{gathered}
f(x, \lambda)=\lambda e^{-\lambda x}, x, \lambda>0 \\
f(x, \alpha, \lambda)=\alpha \lambda\left[1-e^{-\lambda x}\right]^{\alpha-1} e^{-\lambda x}, x, \alpha, \lambda>0 \\
f(x, \alpha, \lambda)=2 \alpha \lambda\left(\frac{1-e^{-\lambda x}}{\left(e^{-\lambda x}\right)^{2}}\right) \exp \left[-\left(\frac{1-e^{-\lambda x}}{e^{-\lambda x}}\right)^{2}\right]\left\{1-\exp \left[-\left(\frac{1-e^{-\lambda x}}{e^{-\lambda x}}\right)^{2}\right]\right\}^{\alpha-1}, x, \alpha, \lambda>0
\end{gathered}
$$

To assess the best model from the competitive distributions we use some information criteria, this includes; Akaike Information Criterion (AIC), Bayesian information criterion (BIC), Corrected Akaike Information Criterion $\left(\mathrm{AIC}_{\mathrm{C}}\right)$ and Hannan - Quinn Information Criterion (HQIC). The least value of these criteria implies the best among the distributions that fit data sets, the formulae of these criteria are given as follow;

$$
\begin{aligned}
& A I C=2 k-2 \ln (L) \\
& B I C=2 k \ln (N)-2 \ln (L) \\
& A I C_{C}=A I C+\frac{2 k^{2}+2 k}{N-k-1} \\
& H Q I C=2 k \ln (\ln (N))
\end{aligned}
$$

where $L$ is the value of the likelihood, $\mathrm{N}$ is the number of recorded measurement and $\mathrm{k}$ is the number of estimated parameter.

\subsection{First Data Set}

The first dataset is strengths of $1.5 \mathrm{~cm}$ glass fiber. It was used previously by (Oguntunde et al., 2017) 
Table 2. Strengths of $1.5 \mathrm{~cm}$ glass fiber

\begin{tabular}{lllllllllllll}
\hline 0.55 & 0.74 & 0.77 & 0.81 & 0.84 & 1.24 & 0.93 & 1.04 & 1.11 & 1.13 & 1.30 & 1.25 & 1.27 \\
1.28 & 1.29 & 1.48 & 1.36 & 1.39 & 1.42 & 1.48 & 1.51 & 1.49 & 1.49 & 1.50 & 1.50 & 1.55 \\
1.52 & 1.53 & 1.54 & 1.55 & 1.61 & 1.58 & 1.59 & 1.60 & 1.61 & 1.63 & 1.61 & 1.61 & 1.62 \\
1.62 & 1.67 & 1.64 & 1.66 & 1.66 & 1.66 & 1.70 & 1.68 & 1.69 & 1.70 & 1.78 & 1.73 & 1.76 \\
1.76 & 1.77 & 1.89 & 1.81 & 1.82 & 1.84 & 1.84 & 2.00 & 2.01 & 2.24 & & & \\
\hline
\end{tabular}

Table 3. The information criteria on the strengths of $1.5 \mathrm{~cm}$ glass fiber

\begin{tabular}{|c|c|c|c|c|c|c|}
\hline Models & Estimates & $\mathrm{AIC}$ & $\mathrm{BIC}$ & $\mathrm{AIC}_{\mathrm{C}}$ & $\mathrm{HQIC}$ & $p$-value \\
\hline $\mathrm{E}_{\mathrm{xp}}-\mathrm{X}$ & $\lambda=0.6648$ & 176.611 & 178.738 & 176.677 & 177.446 & $9.278 \mathrm{e}-10$ \\
\hline $\mathrm{E}_{\mathrm{xp}}-\mathrm{E}$ & $\begin{array}{l}\lambda=0.8693 \\
\alpha=1.9726\end{array}$ & 140.457 & 144.712 & 140.661 & 142.128 & $3.204 \mathrm{e}-05$ \\
\hline $\mathrm{B}_{\mathrm{ux}}-\mathrm{E}$ & $\begin{array}{l}\lambda=0.5350 \\
\alpha=1.9286\end{array}$ & 48.2448 & 52.4990 & 48.4482 & 49.9142 & $1.376 \mathrm{e}-05$ \\
\hline $\mathrm{M}_{\mathrm{wl}} \mathrm{E}$ & $\begin{array}{l}\beta=1.8554 \\
\lambda=0.9043\end{array}$ & 37.1853 & 41.4396 & 37.3887 & 38.8556 & 0.01646 \\
\hline
\end{tabular}

\subsection{Second Data Set}

The second data is the strengths of glass fibers data set comprising 63 observations. It was used by (Ishaq and Abiodun 2020).

Table 4. The strengths of glass fibers

\begin{tabular}{llllllllll}
\hline 0.55 & 0.93 & 1.25 & 1.36 & 1.49 & 1.52 & 1.58 & 1.61 & 1.64 & 1.68 \\
1.73 & 1.81 & 2.00 & 0.74 & 1.04 & 1.27 & 1.39 & 1.49 & 1.53 & 1.59 \\
1.61 & 1.66 & 1.68 & 1.76 & 1.82 & 2.01 & 0.77 & 1.11 & 1.28 & 1.42 \\
1.50 & 1.50 & 1.54 & 1.60 & 1.62 & 1.66 & 1.69 & 1.76 & 1.84 & 2.24 \\
0.81 & 1.13 & 1.29 & 1.48 & 1.50 & 1.55 & 1.61 & 1.62 & 1.66 & 1.70 \\
1.77 & 1.84 & 0.84 & 1.24 & 1.30 & 1.48 & 1.51 & 1.55 & 1.61 & 1.63 \\
1.67 & 1.70 & 1.78 & 1.89 & & & & & & \\
\hline
\end{tabular}


Table 5. The information criteria on the strengths of glass fibers

\begin{tabular}{|c|c|c|c|c|c|c|}
\hline Models & Estimates & AIC & BIC & AIC $_{\mathrm{C}}$ & HQIC & $p$-value \\
\hline $\mathrm{E}_{\mathrm{xp}}-\mathrm{X}$ & $\lambda=0.6637$ & 179.661 & 181.804 & 179.726 & 180.504 & $5.497 \mathrm{e}-10$ \\
\hline $\mathrm{E}_{\mathrm{xp}}-\mathrm{E}$ & $\begin{array}{c}\lambda=0.8693 \\
\alpha=1.9726\end{array}$ & 142.814 & 147.099 & 143.014 & 144.499 & $2.63 \mathrm{e}-05$ \\
\hline $\mathrm{B}_{\mathrm{ux}}-\mathrm{E}$ & $\begin{array}{c}\lambda=0.4793 \\
\alpha=1.9710\end{array}$ & 48.4701 & 52.7563 & 48.6701 & 50.1559 & 0.02577 \\
\hline $\mathrm{M}_{\mathrm{wl}}-\mathrm{E}$ & $\begin{array}{c}\beta=1.7950 \\
\lambda=0.9037\end{array}$ & 37.7465 & 42.0328 & 37.9465 & 39.4323 & 0.00258 \\
\hline
\end{tabular}

It can be observed from tables (3) and (5) that the $\mathrm{M}_{\mathrm{wl}} \mathrm{E}$ distribution is more flexible compare to other competing models because it has the least value of information criteria.

\section{Research Findings}

An extension of exponential distribution namely Maxwell - Exponential distribution was proposed by using Maxwell generalized family of distribution. Its statistical properties such as moments, incomplete moments, probability weighted moments and order statistics was discussed. Simulation study was conducted to demonstrate the potentiality of the proposed model estimator. The proposed model was applied to real life data sets, in both data sets it was shown a great flexibility over the competing models.

\section{Conclusion}

A new extension of Exponential distribution called Maxwell - Exponential distribution was proposed using Maxwell generalized family of distributions. Its statistical properties have been discussed. The plot of Maxwell - Exponential density function possesses the pattern of right and left skewed at different values of parameters. The parameters of the study model were estimated using the maximum likelihood method. The Simulation study was carried out to demonstrate the potentiality of proposed model parameters. An application of Maxwell - Exponential distribution to the real - life data sets was carried out. In both given datasets, the proposed model has performed well more than to the other competing models. Therefore the proposed model is a great substitute to the competing models in modeling these datasets concerning the first data (strengths of $1.5 \mathrm{~cm}$ glass fiber) and the second data (The strengths of glass fibers).

\section{Acknowledgements}

We acknowledge the effort of Editor in Chief and his entire team toward publishing of this paper. 


\section{References}

Ahsan-Ul-Haq, M., \& Elgarrhy, M. (2018). The Odd Frechet-G family of Probability Distributions. $J$. Stat. Appl. Probab. 1(7):185-201.

Alexander, G. N. (1962). The Use of Gamma Distribution in Estimating Regulated Output from Storage Transaction in Civil Engineering. Institute of Engineers, Australia. 4: 29-34,

Alizadeh, M., Altun, E., Cordeiro, G., \& Rasekhi, M. (2017). The Odd Power Cauchy Family of Distributions: Properties, Regression Models and Applications. J. Stat. Comput. Simul. 84(4): 785807.

Alzaatreh, A., Lee, C., \& Famoye, F. (2013). A New Method for Generating Families of Continuous Distributions. Metron. 71(1):63-79.

Bourguignon, M., Silva, R.B., \& Cordeiro, G.M. (2014). The Weibull-G Family of Probability Distributions. J. Data Sci. 12(1): 53-68.

Cordeiro, G.M., Alizadeh, M., Ozel, G., Hosseini, B., Ortega, E.M.M., \& Altun, E. (2017). The Generalized Odd Log-logistic Family of Distributions: Properties, Regression Models and Applications. J. Stat. Comput. Simul. 87(5): 908-932.

Gomes, S. F., Percontini, A., de Brito E., Ramos, M.W., Venancio, R., \& Cordeiro, G. (2017). The Odd Lindley-G family of Distributions. Austrian J. Stat. 46: 65-87.

Gupta, R.D., \& Kundu, D. (2001). Exponentiated Exponential Family, an Alternative to Gamma and Weibull Distributions. Biometrical Journal 1: 117-130.

Ishaq, A.I., \& Abiodun, A. A. (2020). The Maxwell-Weibull Distribution in Modeling Lifetime Datasets. Ann. of Data Sci. 7: 639-662.

Jackson, O. A. Y. (1963). Fitting a Gamma or Log-Normal Distribution to Fiber Diameter Measurement of Wool Tops. Applied Statistics 1: 161-166.

Jamal, F., Nasir, M.A., Tahir, M.H., \& Montazeri, N.H. (2017). The Odd Burr-III Family of Distributions. J. Stat. Appl. Probab. 6(1): 105-122.

Johnson, N.L., Kotz, S. \& Blackrishnan, N. (1994). Continuous Univariate Distribution 1. $2^{\text {nd }}$ edition John Wiley and son, New York.

Klinken, J. \& Van, A. (1961). Method for Inquiring Whether the Gamma Distribution Represent the Frequency Distribution of Industrial Accident Cost. Actualele Studien 3: 83-92.

Muhammad, A., Laba, H., \& Subrata, C. (2018). The Odd Moment Exponential Family of Distributions: its Properties and Applications. Int. J. Appl. Math. Stat 57(6): 47-62.

Nadia, H.A., \& Lamyaa, K.H. (2018). Weighted Exponential-G Family of Probability Distributions. Saudi J. Eng. Technol. 3(2): 51-59.

Oguntunde, P.E., Balogun, O.S.,Okagbue, H.I., \& Bishop, S.A. (2015). The Weibull - Exponential Distribution: Its Properties and Applications. J. of Applied Sciences. 15: 1305-1311 
Oguntunde, P.E., Khaleel, M.A., Adejumo, A.O., Okagbue, A.A., Owalabi, F.O. (2018). The Gompertz Inverse Exponential (GoIE) Distribution with Applications. Cogent Mathematics and Statistics. $5: 1$

Oluyede, B. (2018). The Gamma - Weibull G Family of Distributions with Application. Austrian Journal of Statistics. 47(1): 45-76.

Suleiman, A.A., Ibrahim, A., \& Abdullahi, U.A. (2020). Assessment Probability Distributions of Groundwater Quality Data in Gwale Area, North-Western Nigeria. Annals of Optimization Theory and Practice. 3(1):37-46, doi: 10.22121/aotp.2020.243381.1039.

Weibull, W. (1939). Statistical Theory of the Strength of Materials. Ingenioor Vetenskps Akademiens Handlingar. 151:1-45.

Yousof, H.M., Afify, A.Z., Hamedani, G.G., \& Aryal G. (2016). The Burr X Generator of Distributions for Lifetime Data. J.Stat. Theory Appl. 16:288-305. 